\title{
Central, Extraventricular and Atypical Neurocytomas: a Clinicopathologic Study of 35 Cases from Pakistan Plus a Detailed Review of the Published Literature
}

\author{
Zubair Ahmad, Nasir Ud Din*, Aisha Memon, Mohammad Usman Tariq, \\ Romana Idrees, Sheema Hasan
}

\begin{abstract}
Background: Central neurocytomas are rare neuronal neoplasms with a favorable prognosis. They are typically located in the lateral ventricles of the brain and mostly histologically correspond to WHO grade II with a Mib 1 labelling index of $<2 \%$. Similar tumors located in the cerebral hemispheres and spinal cord, for example, are called "extraventricular neurocytomas". A few tumors histologically show atypia, mitoses, vascular proliferation and/or necrosis and a Mib 1 index $>2 \%$ and are designated as "atypical neurocytomas. Aim: The aim of our study was to describe the common as well as unusual morphologic features and the role of various immunohistochemical stains in the diagnosis of these rare tumors. Materials and Methods: We retrieved and reviewed 35 cases diagnosed between 2001 and 2015. Results: Sixty percent of patients were males, and the mean age was 26 years. 31 cases $(88.6 \%)$ were intraventricular and $4(11.4 \%)$ were extraventricular. Histologically, 6 cases $(17.1 \%)$ were compatible with "atypical neurocytomas". All cases showed the classic morphology comprising nests and sheets of uniform, round cells with uniform round to oval nuclei with finely speckled chromatin and perinuclear cytoplasmic clearing (halos). All cases also showed delicate, fibrillary, neuropil-like matrices. Other common histologic features included capillary-sized blood vessels in a branching pattern in $\mathbf{5 7 . 1 \%}$, foci of calcification in $\mathbf{3 4 . 3 \%}$ and perivascular pseudorosettes in $20 \%$. Rare findings included HomerWright or true rosettes in $8.6 \%$ and ganglioid cells in $2.9 \%$. Synaptophysin was the most consistent and valuable marker, being positive in almost all cases. GFAP positivity in tumor cells was seen in $25.7 \%$ of cases. Follow up was available in 13 patients. Of these 9 had histologically typical and 4 had atypical tumors. Only 1 (with an atypical neurocytoma) died, probably due to complications of surgery within one month, while 12 (including 3 with atypical neurocytomas) remained alive. Recurrence developed in 1 of these 12 patients (histologically consistent with typical morphology) almost 9 years after surgery. Only 4 patients, including 2 with atypical tumors, received postoperative radiotherapy, all with surgery in 2010 or later. Overall, prognosis was excellent with prolonged, recurrence free survival and most patients, even without receiving radiation therapy, were alive and well for many years, even a decade or more after surgery, without developing any recurrence, indicating the benign nature of these neoplasms.
\end{abstract}

Keywords: Central neurocytoma - intraventicular - extraventricular - typical - atypical - synaptophysin

Asian Pac J Cancer Prev, 17 (3), 1565-1570

\section{Introduction}

Gliomas are the commonest central nervous system (CNS) neoplasms worldwide including Asia and Africa (Ahmad et al., 2010; Trabelsi et al., 2014; Zahir et al., 2014). Central Neurocytoma is a rare tumor with a favorable prognosis and is composed of uniform, small round cells with neuronal differentiation. It is typically located in the lateral ventricles of the brain especially near the foramen of Monro but can also be located in the third and even fourth ventricles, occurs mostly in young adults and histologically corresponds to WHO Grade II (Dodero et al., 2000; Figarella-Branger et al., 2007). Extraventricular tumors with a similar histology and located in the cerebral hemispheres or spinal cord are called "extraventricular neurocytomas" or EVNs (Figarella et al., 2007; Brat, 2010). EVNs need to be distinguished from the usual more common oligodendrogliomas which have a relatively worse prognosis (Atalay et al., 2015). The tumor cells are mature neurons which resemble neurocytes similar to those normally found in the dentate fascia of hippocampus and in the cerebellum and were first described as ' neuronal' by Hassoun et al in (Hassoun et al., 1993). In the past, the intraventricular central neurocytomas were often confused with other intraventricular tumors especially ependymomas, oligodendrogliomas, subependymomal astrocytomas etc. However, immunohistochemistry has greatly improved

Department of Pathology and Laboratory Medicine, Aga Khan University Hospital, Karachi, Pakistan *For correspondence: nasir.uddin@aku.edu 
the diagnostic accuracy (Conrad et al., 2000). The tumor cell nuclei have finely speckled chromatin and cytoplasm is clear. Thus these tumors need to be distinguished from other clear cell tumors (Bonney et al., 2015). Central neurocytomas usually have Mib 1 (ki 67) labeling index of less than $2 \%$. Some central neurocytomas have an index higher than $2 \%$, histologically show mitoses, vascular proliferation and/or necrosis, and demonstrate a significantly shorter recurrence free interval. Such tumors are called "atypical central neurocytomas" (Soylemezoglu et al., 1997; Chou et al., 1999; Mackenzie, 1999). However, so far, such atypical lesions have not been assigned a higher WHO grade. The same histologic criteria and Mib 1 labeling index threshold can also be used to designate extraventricular lesions-atypical EVNs (Brat, 2010). Most cases reported in literature including extraventricular and atypical neurocytomas are single case reports or case series involving two or more cases. The aim of this study is to present the cases of Central (and extraventricular) Neurocytomas diagnosed in our department, the largest center for histopathology in Pakistan, over a period of almost 15 years. Our department is a major referral center for challenging and difficult cases covering all subspecialties including brain tumors, from all over Pakistan. We hope that this series of cases will be an important addition to the available literature on these rare tumors.

\section{Materials and Methods}

Slides of all cases reported as Neurocytomas between Jan 1, 2001 and June 30, 2015 in the Section of Histopathology, Department of Pathology and Laboratory Medicine, Aga Khan University Hospital, Karachi were retrieved and reviewed by the two principal authors (ZA and NU). All cases were fixed in $10 \%$ buffered formalin and embedded in paraffin wax. Sections were cut at thickness of 5 microns and were routinely stained with Hematoxylin and Eosin (H\&E). Five microns thick unstained sections were also cut from paraffin blocks for performing Immnunohistochemistery (IHC). These unstained sections were deparaffinized in xylene, hydrated in decreasing concentrations of alcohol solution and then washed in Tris Buffer. IHC staining was performed using avidin-biotin-peroxidase method (Sigma, St Louis, MO). IHC antibodies used included Glial Fibrillary Acidic Protein (GFAP) polyclonal, ready to use -Dako Corporation, (Carpenteria, California; Synaptophysinmonoclonal, Ready to use, Dako Cytomation, Denmark;
Mib 1 (Ki 67)- monoclonal, ready to use, Dako cytomation, Denmark, in most cases. In selected cases, Chromogranin A, monoclonal, ready to use, Dako cytomation, Denmark; and Cytokeratin AE1/AE3 (Monoclonal, ready to use, Dako cytomation), Denmark were also used. All data was analyzed using SPSS 19.0 version software package.

\section{Results}

A total of 35 cases of Central Neurocytoma were reported during the study period (2001-2015). Out of 35,21 patients $(60 \%)$ were males while $14(40 \%)$ were females. Male to fem ale ratio was 1:5:1. Age range was 9 to 51 years. Mean and median age was 26 and 27 years respectively. Out of 35 cases, 31 (88.6\%) were intraventricular, while $4(11.4 \%)$ were extraventricular. Of the 31 intraventricular tumors, $29(93.5 \%)$ were located in the lateral ventricles and $2(6.5 \%)$ were located in the third ventricle. The 4 extraventricular tumors were located in the corpus callosum, thalamus, left temporoparietal hemisphere and the suprasellar region respectively. Histologically, 6 out of 35 (17.1\%) were compatible with "atypical central neurocytoma." Of these, 5 cases were intraventricular in location while 1 case was located in the corpus callosum. The other 29 tumors $(82.9 \%)$ were all histologically compatible with the usual grade II central neurocytomas. Histologically, all cases showed nests and/or sheets of uniform round cells with uniform round to oval nuclei having finely speckled chromatin,

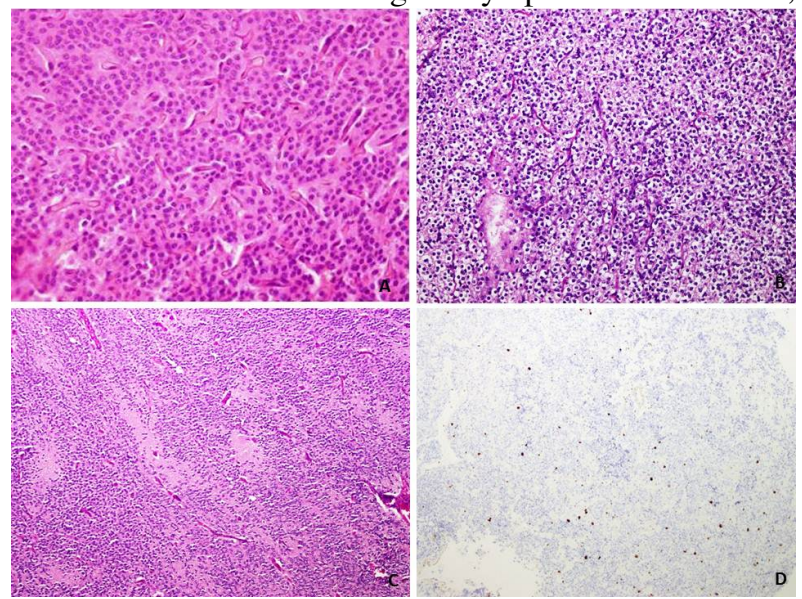

Figure 1.A) Typical Central neurocytoma exhibiting uniform small cells with intervening chicken wire vessels (H\&E, 400x magnification) and $B$ ) perinuclear halos (H\&E, 400x magnification). C) Foci of nuclear free zones (H\&E, 200x magnification). The Ki 67 index is less than $2 \%$ (D)

Table 1. Atypical Neurocytomas in Our Study (n=6)

\begin{tabular}{cccccc}
\hline $\begin{array}{c}\text { Serial } \\
\text { Number }\end{array}$ & Location & $\begin{array}{c}\text { Year of } \\
\text { surgery }\end{array}$ & Mitoses/10HPFs & Mib 1(Ki 67) & Follow up \\
\hline 1 & Intraventricular & 2007 & 3 & $2-5 \%$ & Not available \\
2 & Extraventricular (suprasellar) & 2010 & $>3$ & $>5 \%$ & Died within a month after surgery \\
3 & Intraventricular* & 2010 & 3 & $>5 \%$ & Not available \\
4 & Intraventricular & 2012 & 3 & $>5 \%$ & Alive and well. No recurrence \\
5 & Intraventricular** & 2015 & $>3$ & $>5 \%$ & Alive and well. No recurrence \\
6 & Intraventricular* & 2015 & Alive and well. No recurrence &
\end{tabular}

*Showed vascular proliferation on histological examination; ** Showed necrosis on histological examination 
Central, Extraventricular and Atypical Neurocytomas: a Clinicopathologic Study of 35 Cases from Pakistan Table 2. Immunohistochemical Staining in Our Cases $(n=35)$

\begin{tabular}{ccccc}
\hline Serial number & Immunohistochemical stain & Positive & Negative & Not performed \\
\hline 1 & Synaptophysin & $31(86.6 \%)$ & $1(2.9 \%)$ & $3(8.6 \%)$ \\
2 & GFAP & $9(25.7 \%)$ & $21(60 \%)$ & $5(14.3 \%)$ \\
3 & S 100 protein & $6(17.1 \%)$ & $1(2.9 \%)$ & $28(80 \%)$ \\
4 & Chromogranin A & $8(22.8 \%)$ & $8(22.8 \%)$ & $19(54.3 \%)$ \\
5 & Neuron specific enolase (NSE) & $8(22.8 \%)$ & $2(5.8 \%)$ & $25(71.4 \%)$ \\
6 & CD 56 & $3(8.6 \%)$ & $1(2.9 \%)$ & $31(88.6 \%)$ \\
7 & Neurofilament (NF) & - & $3(8.6 \%)$ & $32(91.4 \%)$ \\
8 & CD 99 (Mic 2) & - & $8(22.8 \%)$ & $27(77.2 \%)$ \\
9 & Epithelial membrane antigen & $3(8.6 \%)$ & $9(25.7 \%)$ & $23(65.7 \%)$ \\
10 & (EMA) & - & $9(25.7 \%)$ & $26(74.3 \%)$ \\
\hline
\end{tabular}

Table 3. Follow Up of Patients in Our Series $(n=13)$

\begin{tabular}{ccccccc}
\hline Serial Number & Year of surgery & Histology & Location & $\begin{array}{c}\text { Radiation } \\
\text { treatment }\end{array}$ & Recurrence & Follow up \\
\hline 1 & 2002 & Typical & Ventricular & No & No & Alive \\
2 & 2003 & Typical & Ventricular & No & No & Alive \\
3 & 2004 & Typical & Ventricular & No & No & Alive \\
4 & 2006 & Typical & Ventricular & No & Yes, in 2015 & Alive \\
5 & 2009 & Typical & Ventricular & No & No & Alive \\
& 2010 & Atypical & Extraventricular & No & No & Died within a \\
6 & 2010 & Typical & Extraventricular & Yes surgery \\
7 & 2010 & Atypical & Ventricular & No & No & Alive \\
8 & 2012 & Atypical & Ventricular & Yes & No & Alive \\
9 & 2012 & Typical & Ventricular & Yes & No & Alive \\
10 & 2012 & Typical & Extraventricular & No & No & Alive \\
11 & 2015 & Typical & Ventricular & No & No & Alive \\
12 & 2015 & Atypical & Ventricular & Yes & No & Alive \\
\hline
\end{tabular}

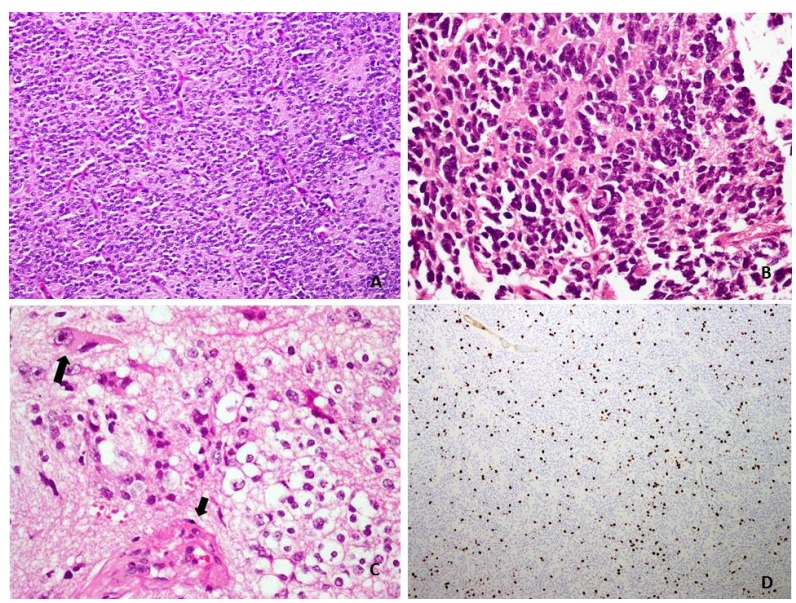

Figure 2.A) An atypical central neurocytoma exhibiting increased cellularity (H\&E, 200x magnification). B) Foci showing cytologic atypia and $\mathrm{C}$ ) a single focus of vascular proliferation is seen in small $\operatorname{arrow}(\mathrm{H} \& \mathrm{E}$, 400x magnification). Increased Ki 67 index (>10\%) is seen $(\mathrm{D})$

inconspicuous nucleoli, and perinuclear cytoplasmic clearing (perinuclear halos). All cases showed delicate, fibrillary neuropil like matrix (Figure 1A-D). Among other histological features, the 6 "atypical" cases demonstrated cellular atypia, increased mitotic activity and Mib 1 (Ki 67) labeling indices above $2 \%$ (Figure 2A-D). However, 3

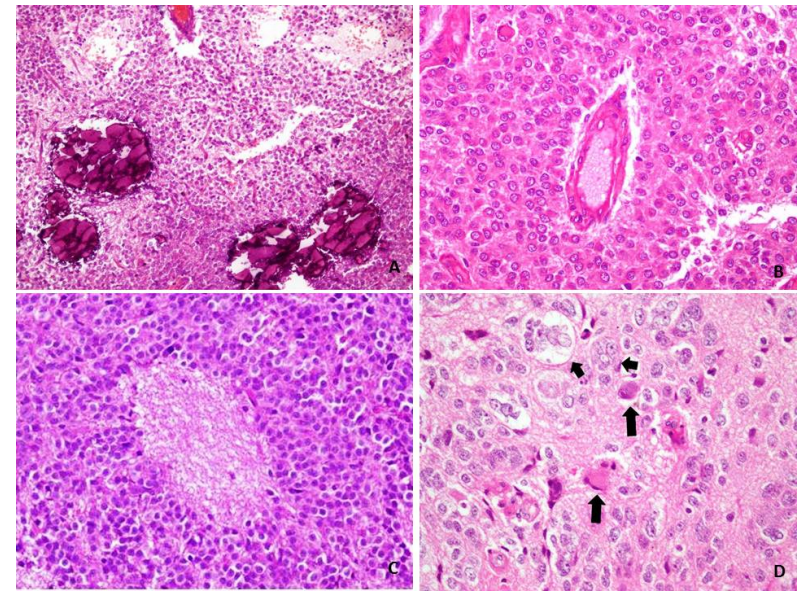

Figure 3. A) Foci of coarse calcification in central neurocytoma (H\&E, 200x magnification). B) Focus of perivascular and C) Homer Wright rosettes . D) Scattered ganglioid cells (large arrow) and multinucleated giant cells (small arrow) $[\mathrm{H \& E}, 400 \mathrm{x}$ magnification]

of the 6 showed $>3$ mitoses/10HPF while 4 of the 6 showed Mib 1 labeling indices $>5 \%$ (Table 1). Of these 6 atypical neurocytomas, 3 showed microvascular proliferation and 1 case also showed necrosis (Figure 2C). These were the only cases in the entire series which showed microvascular proliferation and/or necrosis. Scattered mitotic activity 
and mild or focal atypia were seen in another 8 cases. Hence, a total of 14 out of 35 cases $(42.9 \%)$ showed at least focal atypia and scattered mitotic activity. Capillary sized vessels, arranged in many cases in a branching or arborizing pattern were seen in 20 cases $(57.1 \%)$. Foci of calcification (Figure 3A) were seen in 12 out of 35 cases $(34.3 \%)$. Less frequent histologic findings included perivascular pseudorosettes (Figure 3B) seen in 7 cases (20\%), Homer Wright (Figure 3C) or true rosettes seen in 3 cases (8.6\%), and ganglioid cells (Figure 3D) seen in only 1 out of the total 35 cases $(2.9 \%)$.

In $19(54.3 \%)$ out of 35 cases, the only clinical details provided were those of an intraventricular tumor/mass. In the remaining 16 cases, the clinical signs and symptoms included headache (in 12(34.3\%) cases), vomiting (in 3 cases), seizures (in 2 cases), urinary and/or fecal incontinence (in 2 cases), difficulty in walking (in 2 cases) and personality changes (in 2 cases). The following signs and symptoms were seen in 1 case each: decrease in vision, diplopia, papilledema, vertigo and pain in lower limb.

On immunohistochemistry, synaptophysin was the most consistent and reliable marker being positive in almost all cases in which it was performed. GFAP was mostly negative but showed focal positivity in tumor cells in some cases. The details of immunohistochemical stains analyzed in our cases are shown in Table 2.

Follow up was unfortunately available in only 13 out of 35 cases $(37.1 \%)$. The details are shown in Table 3 .

\section{Discussion}

The incidence rate of Central Nervous System (CNS) neoplasms has risen more than $40 \%$ in the last two decades (Zahir et al., 2014). Central neurocytomas are rare in our practice and composed about $0.7 \%$ of all CNS neoplasms in a study carried in our department (Ahmad et al., 2010). In our series of 35 patients, $60 \%$ were males, mean age was 26 years, $88.6 \%$ tumors were intraventricular, and $93.5 \%$ of intraventricular tumors were located in the lateral ventricles. There were 4 extraventricular tumors comprising $11.4 \%$ of the total while $6(17.1 \%)$ were histologically compatible with atypical neurocytomas with Mib 1 (ki 67) levels greater than 2\%. Only 1 of the 6 atypical neurocytomas was extraventricular. Studies have shown that this tumor affects males and females almost equally, and mainly affects young adults. Mean age was 29 years in one study (Tacconi et al., 1997; Conrad et al., 2000; Figarella-Branger et al., 2007). Tacconi et al published a series of 5 cases in 1997 and stressed the importance of diagnostic suspicion, total surgical excision and radiotherapy in the prognosis of this tumor (Tacconi et al., 1997). In the year 2000, a number of studies were published on central neurocytomas. Conrad et al published a clinicopathological analysis of 3 cases in which they discussed the clinical and radiological features, surgical findings and pathological appearance of this tumor (Conrad et al., 2000). The same year Dodero et al reported 2 cases of 'giant' central neurocytomas in two young women with both cases showing triventricular extension (Dodero et al., 2000). In a series of 10 cases, Favereaux et al, also in 2000, discussed the histopathological variants of central neurocytoma. In their series, 2 out of 10 cases fulfilled the histological criteria for atypical neurocytomas. These 2 cases showed less marked reactivity for the neuronal marker Synaptophysin compared to the 8 "classical" cases. Their 2 atypical cases were cellular with increased mitotic activity. One case also showed tumor necrosis. Both their atypical cases had Mib 1 labeling index $>5.2 \%$ and both tumors recurred. Based on this, they concluded that high mitotic activity, necrosis, loss of neuronal differentiation on immunohistochemistry and high Mib 1 labeling index were the histological and immunohistochemical criteria for aggressive behavior (Favereaux et al., 2000). Chou et al reported a case of atypical neurocytoma. The histology in their case showed up to 3 mitoses/10HPF and Mib 1 labeling index was 5\%. Their patient died of hydrocephalus and brain edema the day after he underwent partial resection. They noted that atypical neurocytomas require postoperative radiotherapy (Chou et al., 1999). In a comprehensive review published in 2006, Sharma et al discussed the epidemiology, histogenesis, clinical presentation, the characteristic neuroimaging and histopathologic findings and the therapeutic options. They concluded that safe maximal resection is the best therapeutic option and shows the best long term results when taking into account the local control of the tumor and long term survival of the patient. They emphasized the beneficial effect of adjuvant radiotherapy in atypical neurocytomas and when the tumor is incompletely resected (Sharma et al., 2006). Ulivieri and Oliveri described a single case in a 25 year old male in 2007 (Ulivieri and Oliveri, 2007). However, two relatively large series (of 9 cases each) were published in 2008. The first of these published by Chen et al demonstrated high proliferative index (Mib 1 labeling index upto 6.8\%) in two patients in whom the disease recurred. They also showed that apart from high proliferation index, vascular proliferation and synaptophysin expression were more prominent in the recurrent tumors compared to the primary. However, they concluded that recurrent tumors are usually local and patients undergo full recovery following a second surgical resection and radiotherapy (Chen et al., 2008). This indicates that Mib 1 ( $\mathrm{Ki} \mathrm{67)} \mathrm{labeling} \mathrm{index} \mathrm{is} \mathrm{a}$ valuable prognostic factor in Neurocytomas, and not just in gliomas, where its role as a prognostic factor is well established (Chen W-T et al., 2015). The other study by Chen et al, which also looked at nine cases, followed the patients for 8 to 33 months after surgery and used various methods to assess the functional outcomes of central neurocytomas. They found that most of the patients achieved good functional outcomes and showed good ability to perform their daily activities independently. They recommended that although these tumors are mostly benign, patients should be regularly followed up in order to detect any recurrence of the tumor (Chen et al., 2008).

A multidisciplinary review by Chodhari et al published in 2009 concluded that recurrence rate for central neurocytomas after surgery was more common than previously thought. The authors therefore recommended a multi-modality team involvement for optimum management of these tumors (Chodhari et al., 2009). Chen et al published a series of ten cases in 2012, nine 
of which were located in the lateral ventricles while one case was extraventricular (in the sellar region). Following surgical resection, six patients (four with subtotal and two with total resection) received radiotherapy. None of the tumors in their series recurred (Chen et al., 2012). Kane et al published a remarkably large series in 2012 solely on intracranial extraventricular neurocytomas (EVNs). This series comprised 85 patients of whom $27 \%$ had atypical EVNs on histologic examination. The authors demonstrated the better prognosis of typical EVNs compared to atypical EVNs following surgical resection. According to their findings typical EVNs had a 5 -year recurrence rate of $36 \%$ and a 5 year mortality rate of $4 \%$ compared to 5 year recurrence rate of $68 \%$ and 5 year mortality rate of $44 \%$ for atypical EVNs. The difference was statistically significant $(\mathrm{p}<0.001)$. They also found that for both typical and atypical EVNs, gross total resection was more beneficial compared to subtotal resection and that radiotherapy (in both typical and atypical EVNs) benefited patients with subtotal resection of the primary tumor (Kane et al., 2012). Canova at al noted that the main treatment of central neurocytomas which is total resection is achieved in only half the cases. They argue that external beam radiotherapy was beneficial in patients with subtotal resection as well as in those with atypical central neurocytomas. They also discussed the role of chemotherapy in patients with extracranial and/or neuromeningeal spread of the tumor, and in tumors which recurred after surgery and/or radiotherapy (Canova at al., 2014). In a recent study published in 2015, Bonney et al looked at the molecular biology of central neurocytomas and acknowledged that although progress on this front was being made, this had not yet translated into the development of targeted therapies for these tumors (Bonney et al., 2015). A recent 2015 article by Yang et al focused on the clinical manifestations of these tumors and concluded that although no clinical feature was pathognomonic, patients typically presented with signs and symptoms of increased intracranial pressure (Yang et al., 2015). Other authors also agree that manifestations of increased intracranial pressure usually brought these tumors to clinical attention (Chen et al., 2008; Chen et al., 2012; Canova et al., 2014). Kawano et al recently reported a case of atypical EVN in a 3 year old girl. Atypical EVN in children are extremely rare. However, the authors argue that atypical EVN should be considered in the differential diagnosis of an intracranial but extraventricular cystic tumor in children (Kawano et al., 2015).

In a comprehensive review on extraventricular neurocytomas, published in 2014, Patil et al discussed the clinical and pathologic features of these tumors, the effectiveness of surgical resection and progression free survival. Of their seven cases, ages 7 to 65 years, four were located in the cerebral hemispheres, and 1 each in the cerebellum, pineal gland and spinal cord. They found that gross total resection and subtotal resection plus radiotherapy had similar outcomes, while extremes of age and atypical histology adversely affected the prognosis (Patil et al., 2014). A recent article by Sweiss et al also reviewed EVNs including their epidemiology, clinical and radiological findings, locations, treatment and prognosis and concluded that being quite rare, the epidemiology of EVNs was not definitely known and they were associated with a poor prognosis compared to central neurocytomas. They argued that effective treatment strategies for these rare tumors were still lacking (Sweiss et al., 2015).

The detailed discussion above clearly highlights the importance of adjuvant radiotherapy in the treatment of both central and extraventricular neurocytomas especially in the presence of subtotal resection. Various studies discussed above clearly demonstrate that radiotherapy increases local control of the tumor. However, a study by Vrana et al in 2013, while acknowledging the usefulness and importance of adjuvant radiotherapy in neurocytomas cautioned that the timing of radiotherapy in these patients needed to be considered carefully to protect these patients from possible late side effects of radiotherapy since most patients with neurocytomas have long time survival following treatment (Vrana et al., 2013).

In addition to the usual microscopic findings, rare features such as Homer Wright and true rosettes and ganglioid cells were seen in 3 and 1 of our 35 cases respectively. These rare features have been described in these tumors (Robbins et al., 1995).

Synaptophysin is the most reliable immunohistochemical marker in these tumors and diffuse positivity is seen in the neuropil, the fibrillary zones and perivascular nuclear-free zones (Figarella-Branger et al., 1992). Synaptophysin was also the best marker in our series being positive in almost all cases in which it was performed. Neu $\mathrm{N}$ and anti-HU antibodies are also considered to be very useful and demonstrate nuclear staining in most cases (Gultekin et al., 1998; Soylemezoglu et al., 2003). Unfortunately, we do not have Neu N and anti-HU antibodies in our immunohistochemistry panel as there are issues of financial feasibility. GFAP staining has been mostly reported in trapped reactive astrocytes, however some authors have reported GFAP positivity in tumor cells (von Deimling et al., 1990). As shown in the results, GFAP positivity was seen in focal cells in over $25 \%$ of our cases. Chromogranin A and Neurofilament are usually negative in central neurocytomas (Hassoun et al., 1993). Neurofilament was performed in only 3 of our cases and was negative in all 3. However, chromogranin A which was performed in almost $45 \%$ of our cases was positive in half of these. It also needs to be noted that diffuse, widespread expression of the immunohistochemical stain OLIG 2 helps in differentiating central neurocytomas (usually negative or focally positive in a minority of cases) and clear cell ependymomas from oligodendrogliomas (widespread OLIG 2 expression in almost all cases (Preusser et al., 2007).

Follow up was available in only 13 out of 35 cases. This is unfortunate but perhaps inevitable considering that we receive cases from all over Pakistan, a country of 180 million people, and many cases come from far off and remote areas. A glance at Table 3 shows that 12 out of 13 patients (in whom follow up was available) were alive at the time of follow up. Of the 4 patients with atypical neurocytomas (in whom follow up was available), 1 patient died within a month of surgery (in 2010). This tumor was extraventricular and the patient 
most likely died of complications arising after surgery. Of the other 3 patients (operated in 2010, 2012 and 2015), 2 received postoperative radiation, all 3 were alive with no recurrence. Of all 13 patients, only 1 patient with an intraventricular typical central neurocytoma recently developed recurrence (in 2015), almost 9 years after surgery in 2006. This patient had not received any radiation treatment following initial surgery. Overall, out of 13 , only 5 patients received radiation (all with surgery in 2010 or later). However, as shown in Table 2, patients with initial surgery as far back as 2002 and 2003, who did not receive any radiation treatment, are all alive and well at the time of recent follow up. The 3 patients with 'atypical' neurocytomas also were alive and well at follow up. All had surgery witin the last three years ( 2 of them in 2015). They need to be followed for a longer time to determine their clinical behavior.

In conclusion, we present a series of 35 cases of neurocytoma including extraventicular and atypical neurocytomas that were diagnosed in our department over a period of almost 15 years. We hope that our series will be an important addition to the available literature on these rare tumors.

\section{References}

Ahmad Z, Arshad H, Hasan S, et al (2010). CNS neoplasms in Pakistan, a pathological perspective. Asian Pac J Cancer Prev, 12, 317-21.

Atalay T, Ak Hakan, Celik B, et al (2015). Prognostic factors in oligodendrogliomas: a clinical study of twenty-five consective patients. Asian Pac J Cancer Prev, 16, 5319-23.

Bonney PA, Boettcher LB, Krysiak RS 3rd, et al (2015). Fung $\mathrm{KM}$, Sughrue ME. Histology and molecular aspects of central neurocytoma. Neurosurg Clin N Am, 26, 21-9.

Brat DJ. Neuronal and Glioneuronal Neoplasms. In Perry A, Brat DJ eds (2010). Practical Surgical Neuropathology. A diagnostic approach . Churchill Livingstone Elsevier Inc Philadelphia, PA, 125-50.

Canova CH, Riet FG, Idbaih A, et al (2014). Radiotherapy in central neurocytoma and review of literature. Cancer Radiother, 18, 222-8.

Chen CL, Shen CC, Wang J, et al (2008). Central neurocytoma: a clinical, radiological and pathological study of nine cases. Clin Neurol Neurosurg, 110, 129-36.

Chen CM, Chen KH, Jung SM, et al (2008). Central neurocytoma: 9 case series and review. Surg Neurol, 70, 204-9.

Chen H, Zhou R, Liu J, et al (2012). Central neurocytoma. J Clin Neurosci, 19, 849-53.

Chen WJ, He DS, Tang RX, et al (2015). Ki-67 is a valuable prognostic factor in gliomas: evidence from a systematic review and meta-analysis. Asian Pac J Cancer Prev, 16, 411-20.

Chou YY, Lee CC, Chen TJ, et al (1999). Atypical central neurocytoma: report of a case. J Formos Med Assoc, 98, 573-7.

Choudhari KA, Kaliaperumal C, Jain A, et al (2009). Central neurocytoma: a multi-disciplinary review. Br J Neurosurg, 23, 585-95.

Conrad MD, Morel C, Guyotat J, et al (2000). Central nervous system neurocytomas: clinicopathological analysis of tree cases. Arq Neuropsiquiatr, 58, 1100-6.

Dodero F, Alliez JR, Metellus P, et al (2000). Central neurocytoma: 2 case reports and review of the literature. Acta Neurochir (Wien), 142, 1417-22.
Favereaux A, Vital A, Loiseau H, et al (2000). Histopathological variants of central neurocytoma: Report of 10 cases. Ann Pathol, 20, 558-63.

Figarella-Branger D, Pellissier JF, Daumas-Duport C, et al (1992). Central neurocytomas. Critical evaluation of a small-cell neuronal tumor. Am J Surg Pathol, 16, 97-109.

Figarella-Branger D, Soylemezoglu F, Burger PC (2007). Central Neurocytoma and extraventricular neurocytoma. In Louis DN, Ohgaki H, Wiestler OD, Cavenee WK. WHO Classification of Tumors of the Central Nervous System. 4th Edition. IARC, Lyon, 106-9.

Gultekin SH, Dalmau J, Graus Y, et al (1998). Anti-Hu immunolabeling as an index of neuronal differentiation in human brain tumors: a study of 112 central neuroepithelial neoplasms. Am J Surg Pathol, 22, 195-200.

Hassoun J, Gambarelli D, Grisoli F, et al (1998). Central neurocytoma. An electron-microscopic study of two cases. Acta Neuropathol, 56, 151-6.

Hassoun J, Soylemezoglu F, Gambarelli D, et al (1993). Central neurocytoma: a synopsis of clinical and histological features. Brain Pathol, 3, 297-306.

Kane AJ, Sughrue ME, Rutkowski MJ, et al (2012). Atypia predicting prognosis for intracranial extraventricular neurocytomas. J Neurosurg, 116, 349-54.

Kawano H, Kimura T, Iwata K, et al (2015). Atypical extraventricular neurocytoma in a 3-year-old girl: case report with radiological-pathological correlation. Childs Nerv Syst, 31, 1189-93.

Mackenzie IR (1999). Central neurocytoma: histologic atypia, proliferation potential, and clinical outcome. Cancer, 85, 1606-10.

Patil AS, Menon G, Easwer HV, et al (2014). Extraventricular neurocytoma, a comprehensive review. Acta Neurochir, 156, 349-54.

Preusser M, Budka H, Rossler K, et al (2007). OLIG2 is a useful immunohistochemical marker in differential diagnosis of clear cell primary CNS neoplasms. Histopathol, 50, 365-70.

Robbins P, Segal A, Narula S, et al (1995).Central neurocytoma. A clinicopathological, immunohistochemical and ultrastructural study of 7 cases. Pathol Res Pract, 191, 100-11.

Sharma MC, Deb P, Sharma S, et al (2006). Neurocytoma: a comprehensive review. Neurosurg Rev, 29, 270-85.

Soylemezoglu F, Scheithauer BW, Esteve J, et al (1997). Atypical central neurocytoma. J Neuropathol Exp Neurol, 56, 551-6.

Soylemezoglu F, Onder S, Tezel GG, et al (2003). Neuronal nuclear antigen (NeuN): a new tool in the diagnosis of central neurocytoma. Pathol Res Pract, 199, 463-8.

Sweiss FB, Lee M, Sherman JH (2015). Extraventricular neurocytomas. Neurosurg Clin N Am, 26, 99-104.

Tacconi L, Thom M, Symon L (1997). Central neurocytoma: a clinico-pathological study of five cases. Br J Neurosurg, 11, 286-9.

Trabelsi S, Brahim DH, Ladib M, et al (2014). Glioma Epidemiology in the Central Tunisian Population:1993-2012. Asian Pac J Cancer Prev, 15, 8753-7.

Ulivieri S, Oliveri G (2007). Intraventricular neurocytoma: case report. Pathologica, 99, 309-12.

von Deimling A, Janzer R, Kleihues P, et al (1990). Patterns of differentiation in central neurocytoma. An immunohistochemical study of eleven biopsies. Acta Neuropathol, 79, 473-9.

Vrana D, Cwiertka K, Lukesova L, et al (2013). Patient with atypical neurocytoma -case report. Klin Onkol, 26, 362-4.

Yang I, Ung N, Chung LK, et al (2015). Clinical manifestations of central neurocytoma. Neurosurg Clin N Am, 26, 5-10.

Zahir ST, Vakili M, Navabii H, et al (2014). Clinicopathological findings and five year survival rates for patients with central nervous system tumors in Yazd, Iran. Asian Pac J Cancer Prev, 15, 10319-23. 